\title{
Advanced Measuring (Instrumentation) Methods for Nuclear Installations: A Review
}

\author{
Wang Qiu-kuan, Lin Chang-hua, and Yang Yan \\ Chongqing Automobile College, Chongqing University of Technology, Chongqing 400054, China \\ Correspondence should be addressed to Yang Yan, ahasnake@126.com
}

Received 14 July 2012; Accepted 27 August 2012

Academic Editor: Xing Chen

Copyright ( $) 2012$ Wang Qiu-kuan et al. This is an open access article distributed under the Creative Commons Attribution License, which permits unrestricted use, distribution, and reproduction in any medium, provided the original work is properly cited.

The nuclear technology has been widely used in the world. The research of measurement in nuclear installations involves many aspects, such as nuclear reactors, nuclear fuel cycle, safety and security, nuclear accident, after action, analysis, and environmental applications. In last decades, many advanced measuring devices and techniques have been widely applied in nuclear installations. This paper mainly introduces the development of the measuring (instrumentation) methods for nuclear installations and the applications of these instruments and methods.

\section{Introduction}

In last decade, nuclear technology has developed rapidly and became more important to human society with the development of science and technology. Nuclear technology has many advantages, such as zero carbon emissions, energy independence, and safety. At present, nuclear installations are more prevalent than ever before. Therefore, with the rapid development and wide applications of nuclear technology, many new technologies have been emerging to guarantee its reliability and safety, where measuring devices and techniques that can exactly measure and monitor the nuclear installations show particular importance.

This paper is a review of advances in measuring (instrumentation) technology focus areas that have applications in nuclear installations. The instruments used in nuclear installations mainly include multifarious detectors, sensors, and meters. The measurement methods for nuclear technology mostly consist of all kinds of nondestructive measurement methods. The paper presents various instruments and measurement methods which are widely used in nuclear power reactors, nuclear fuel cycle, safeguards and homeland security, nuclear accident, after action, and analysis.

\section{Nuclear Power Reactors}

2.1. Nuclear Power Reactors Instrumentations. In this section, instrumentations to measuring the neutron fission, the neutron dose, the flux, the reactor fission rate, and temperature are discussed. Here instrumentations contain Micro-Megas detectors, noise thermometer, tissue equivalent proportional counter, high-temperature thermocouples, liquid scintillation detector, spectrometers, optical fiber sensors, Uranium Fission chamber, Plutonium Fission chamber, Self-Powered Neutron Detector, Ionization chamber, SelfPowered Gamma Detector, Differential calorimeter, Gamma Thermometer, and Optical Fiber Sensors, and so forth.

A new set-up at the Conseil Europeen pour la Recherche Nucleaire (CERN) n_TOF facility has been built and tested by Guerrero et al. which allowed measuring simultaneously neutron, induced fission and capture reactions by combining a $4 \pi$ Total Absorption Calorimeter (TAC) with several Micro-Me-gas (MMGAS) detectors [1]. The sketch is shown in Figure 1. Bolshakova et al. discussed the issues of creating the instrumentation for measured the semiconductor magnetic field sensors during their irradiation with neutrons in nuclear reactors up to fluences similar to neutron fluences in 


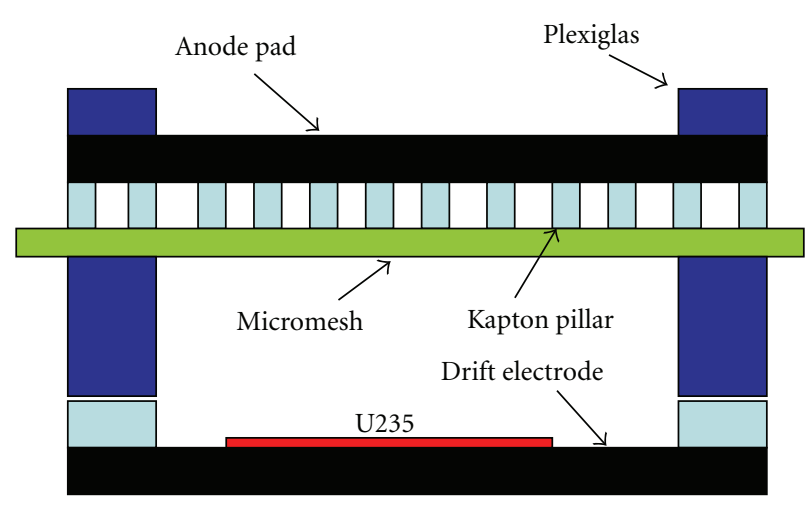

FIGURE 1: Detail of the MMGAS detectors.

steady-state sensor locations in international thermonuclear experimental reactor [2]. They used the tissue equivalent proportional counter to measure the neutron dose and dose equivalent rates, and the multisphere spectrometers were used in measurement of energy distributions [3]. Fourmentel et al. did a lot of work to measure more accurately photon flux, nuclear heating, and neutron flux in the reactor [4]. The devices measure the photon flux by two different sensors (Ionization chamber and Self-Powered Gamma Detector), the nuclear heating by two different ones (Differential calorimeter and Gamma Thermometer), and the neutron flux by three different kinds of sensors (Uranium Fission chamber, Plutonium Fission chamber and Self Powered Neutron Detector).

A key technique in nuclear installations is monitoring of temperature. Brixy used the noise thermometer to measure the temperature in nuclear reactors [5]. The noise thermometer rooted in the Nyquist theorem which is used to determine absolute temperatures. Both of the two resistors have noise voltages, one at the unknown temperature, the other at room temperature. Compared with the ratio of the resistances, when the noise voltages from the two resistors are matching, the ratio of their absolute temperatures is determined. Bily and Sklenka designed a new instrumentation for measurement of thermal effects on the training reactor which called VR-1 [6]. Rempe et al. contrasted the types of sensors available to support in-pile irradiations measurement and those sensors available to Advanced Test Reactor (ATR) currently [7]. Accomplishments from new sensor technology deployment efforts are remarkable by describing new thermal and temperature conductivity sensors available to ATR users now. The sketch is shown in Figure 2.

The major superiority obtained over the last decade in the understanding of fundamental neutrino physics allowed us to put into use the detection of reactor antineutrino signals to automatic and nonintrusive nuclear power plant investigates. Porta et al. presented the NUCIFER experiment, which used a 1-ton Gd-doped liquid scintillator detector to be installed a few $10 \mathrm{~m}$ from a reactor core for measurements of its Plutonium content and thermal power [8]. The design of such a small bulk detector has been focused on good background rejection and high detection efficiency. The advanced qualification of innovative high-temperature

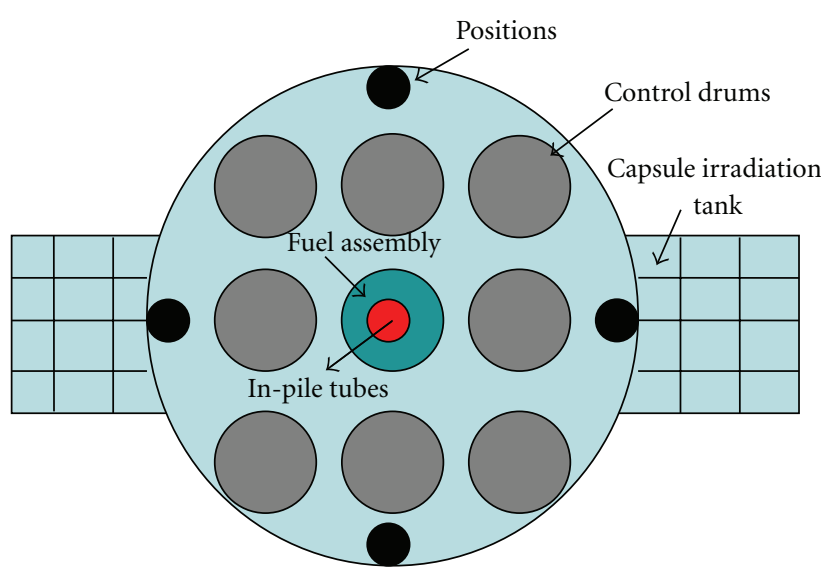

FIgURE 2: The irradiation locations of ATR core cross-section.

thermocouples specifically for in-pile applications was designed by Villard et al. [9]. This new kind of thermocouple was based on niobium thermoelements and molybdenum, which persisted nearly unchanged by thermal neutron flux even though under harsh nuclear environments, whereas Type $\mathrm{C}$ or Type $\mathrm{S}$ as typical high-temperature thermocouples is changed by significant drifts caused by material deformations under the same conditions.

The other key issue for advanced irradiation programs in nuclear research reactors is in situ measurement, especially in Material Testing Reactors (MTR). With the prospective, Optical Fiber Sensors (OFSs) is a good choice. OFS can provide unique feature substantial properties that bring intrinsic advantages over conventional sensing approaches. An initial condition for the development of OFS ensures that the Radiation-Induced Absorption (RIA) of the light in the fiber does not exceed a predefined threshold. Cheymol et al. reported the results of a long-lasting irradiation investigation, which carried out various fibers, including Single Mode (SM) fibers and Multimode (MM) and hollow core Photonic Band Gap (PBG) fibers [10]. for application of a Fiber Optic Fabry-Perot Interferometer (FOFPI) for measurements of the pressure variation in water reactors of nuclear power plants, the intelligent sensors could contribute to improving their safety and long-term metrological stability [11]. The algorithm of self-calibration of the pressure sensor based on the fact that the length of the FOFPI cavity depends on the pressure at a constant spectrum of an optical source is considered. It is shown that manufacturing of an FOFPI-sensitive element based on a molecular layering nanotechnology provides for substantial enhancement of the quality of the gauge.

2.2. Nuclear Power Reactors Measurement Methods. In this section, nuclear power reactors measurement methods are discussed. Here measurement methods contain indium foils methods, solid state nuclear track detection, time-of-flight technique, microwave propagation methods, optical dosimetry, accelerator mass spectrometry, gamma spectrometry and swept wavelength interferometry, and so forth. These measurement methods can be used to measure neutron flux, 
reactor fission rate, absolute energy of cold neutrons, radiation field intensity, electronic excitation dose rate, atomic displacement, thermal neutron flux, and volatile fission.

Papastefanou reported the measurement of albedo of water and neutron flux for thermal neutrons with indium foils and the analyses of multiplication factor, relaxation length, neutron amplification, migration length, and parameters of the nuclear reactor [12]. Li et al. described the measurement principle and method of fission rate of nuclear reactor by solid-state nuclear track detection and showed the measurement result for miniature neutron source nuclear reactor [13]. This method has advantage over other physics method which avoided the approximate supposition of some other method in fission rate measurement. Laurie et al. summed up data collected from previous irradiation tests in terms of thermocouple data [14]. Khentout and Meftah presented a method for measuring the neutron diffraction facility at the Es-Salam nuclear power reactor and the absolute energy of cold neutrons at the small angle scattering facility of the NUR nuclear power reactor using the timeof-flight technique [15]. Bhattacharya et al. reported an investigation of the radiation field intensity measurement in the core of a pulsed nuclear reactor using microwave propagation through an appropriately chosen noble gas placed within the nuclear power reactor [16].

Meirav et al. used the method of accelerator mass spectrometry which is applied to the measurements of concentrations of the long-lived volatile fission product in the Irradiated Fuel Bay (IFB) of a nuclear power reactor and Primary Heat Transport (PHT) system [17]. Coulon et al. used gamma spectrometry to monitor the fourth generation Sodium Fast Reactor (SFR) nuclear power [18]. Simulation showed that power monitoring in high accuracy and with short response time is possible measuring delayed gamma emitters produced in the liquid sodium. Gamma emitter production rate in the reactor core was calculated with technical and nuclear data as atomic densities, neutron spectra, sodium velocity and incident neutron cross-sections of fission reactions, and also sodium activation reactions producing gamma emitters. Sang et al. reported the applications of Swept Wavelength Interferometry (SWI) to distributed fiber-optic temperature measurements in a nuclear reactor, the sensors consisted of 2 segments of single mode optical fibers [19]. The sketch is shown in Figure 3. Yarovoi et al. applied the holographic equipment in diagnosis of NPP construction. There is a possibility of application of interference and holographic technique in condition of NPP [20].

\section{Nuclear Fuel Cycle}

3.1. Nuclear Fuel Cycle Instrumentation. The nuclear fuel cycle consists of steps in the front end, which are the preparation of the fuel, steps in the service period, in which the fuel is used during reactor operation, and steps in the back end, which are necessary to safely manage, contain, and either reprocess or dispose of spent nuclear fuel. The instrumentations can measure nuclear fuel reprocessing, nuclear fuel inpool, spent fuel assembly, the absolute energy-integrated flux and the energy spectrum of anti-neutrinos emitted adopted

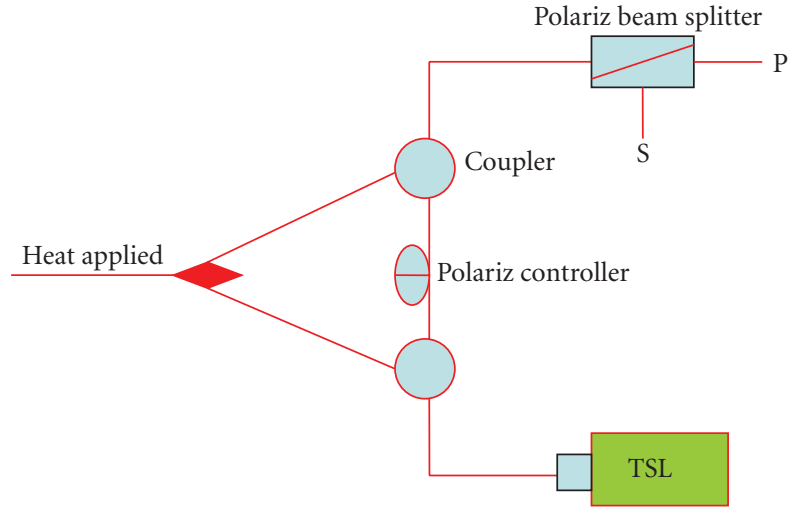

FIGURE 3: Optical network applied for polarization diverse measurement of Rayleigh backscatter.

optical fiber sensor, high-resolution germanium detector, alanine dosimeters, and energy spectrum.

A framework of performance metrics, which coupled with a first-order lumped reactor model to determine nuclide population balances, can be used to quantify the aforementioned cons and pros for a range of different reactor and nuclear fuel cycle combinations [21]. The framework includes metrics such as fuel reprocessing, fuel efficiency, spent fuel toxicity, and proliferation resistance. Nuclear fuel reprocessing requires high acidity levels that should be rapidly and accurately controlled in order to optimize extraction process and yields efficiency [22]. A new optical fiber sensor based on a sol-gel film doped with an acid-sensitive indicator has been developed to improve remote and on-line monitoring of the nuclear fuel cycle. The system is based on a submergible housing, containing a high-resolution germanium detector, allowing for the measurement of nuclear fuel in-pool [23]. The system can be transported to any nuclear power plant's fuel storage pool for in-pool measurements during outage. The measurement of radiation from a spent nuclear fuel assembly is based on usage of passive detectors, such as track detectors for neutron radiation and alanine dosimeters for gamma radiation [24]. Battaglieri et al. presented the expected performance of a new detector to measure the energy spectrum and the absolute energy-integrated flux of antineutrinos emitted by an unclear power plant fuel cycle [25].

3.2. Measurement Methods for Nuclear Fuel Cycle. Another description of the nuclear fuel cycle includes manufacture of reactor fuel element and fuel preparation, nuclear fuel materials cycle, the composition of fuel rod, reprocessing of fast reactor fuel, assaying spent fuel, processing spent fuel, the process of nuclear fuel fabrication and reprocessing. All of these steps can be measured by neutron absorption technique, detection of high energy gammarays emitted methods, DFT methods, nondestructive acoustic method, conduct metric technique, ultrasonic transmission technique, pyrometallurgical technology, nondestructive assay technique, photon gamma spectrometry, and so forth. 


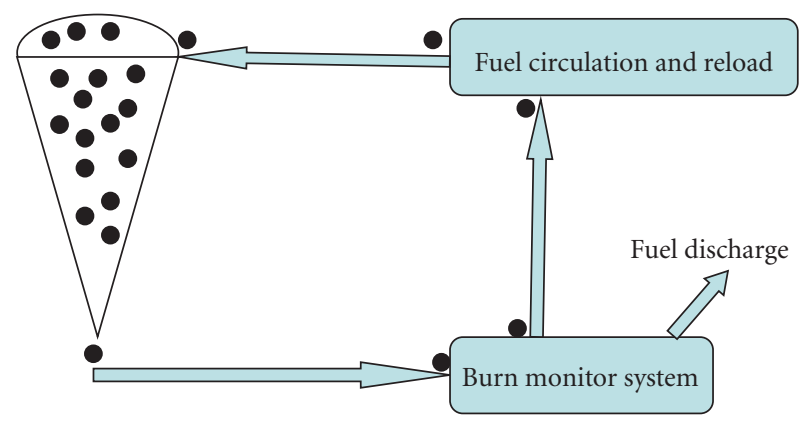

FIgURE 4: The multipass MPBR of fuel circulation.

A nondestructive method based on a neutron absorption technique has been developed for the process-control analysis of ${ }^{235} \mathrm{U}$ in manufacture of reactor fuel element [26]. Perret and Jordan applied a novel technique to measure the pent fuel of fissions after reirradiation at low power [27]. This technique is based on the detection of high energy gamma rays emitted by delayed neutrons and short-lived fission products. Liu et al. reviewed the state of first-principles Density Functional Theory (DFT) modeling of nuclear fuel materials cycle [28]. The first-principles modeling of DFT has emerged as a quantitatively rigorous method which was widely used to study nuclear materials cycle. Rosenkrantz et al. developed a nondestructive acoustic method that gives an easy access to the composition and the pressure of the internal gas mixture in the upper plenum of a standard fuel rod [29]. Subbuthai et al. tested a conductometric technique for determination of the third phase, a phase formed by splitting of organic phase during malfunction in operation [30]. Kamei and Serizawa concerned with a new development of ultrasonic transmission technique for film flow measurements [31]. The technique adopted a rotating reflector, capable of measuring time-dependent spatial distribution of liquid film thickness around a simulated nuclear fuel rod. Inoue proposed pyrometallurgical technology which is one of potential devices for future nuclear fuel cycle [32]. The main technology is electrorefining for $\mathrm{U}$ and $\mathrm{Pu}$ separation, which can be applied on oxide fuels through reduction process as well as metal fuels.

Optical method is important to the measurement of nuclear fuel cycle. Normand et al. introduced a new concept of nuclear instrumentation system [33]. This new measurement system could make measurements in active neutron condition, especially time stamping, as well as photon gamma spectrometry. This development measurement system aims to optimize nuclear fuel cycle. Badawy and EIGammal measured the low enriched uranium fuel cycle pellets for a nuclear reactor by a passive nondestructive assay technique [34]. The method adopted a high-resolution germanium detector gamma-ray spectrometer. It is based on the measurement of a certain characteristic gamma-rays peak of the $\mathrm{U}^{235}$ in the nuclear fuel pellets. Gavron et al. analyzed the potential capability of using lead slowing down spectrometer technology in assaying spent fuel [35]. Das et al. described the development of a spectrophotometric method for trace level determination of U(VI) during the reprocessing and postprocessing of nuclear fuel fabrication in clear industries [36]. Hawari et al. assessed the feasibility of passive gamma-ray spectrometry assay as an approach for on-line burnup determination for nuclear fuel of the Modular Pebble Bed Reactor (MPBR) [37]. The sketch is shown in Figure 4. Meier et al. illustrated the utilizations of optically accessible high-pressure sector test rig in combination with optical test methods as part of the development process of a fuel lean injection combustion concept. Imaging and laserbased techniques are used to characterize fuel preparation, temperature, heat release, and nuclear fuel cycle [38].

\section{Safeguards and Homeland Security}

In this section, many nuclear instrumentations and measurement methods for safe and secure environmental applications will be discussed. Recently, the mainly nuclear instrumentations and measurement methods include Nondestructive Assay (NDA) techniques, Temperature Semiconductor (TS) detector, Na/I detector, High Pressure xenon (HPXe) detector, plastic scintillator, genetic programming algorithm, automated analytical chemistry laboratory instrumentation and tamper-resistant liquid level/accountability instrumentation, system, which can be used for guarantee safeguards and homeland security.

Nuclear safeguards rely, in part, on NDA verification of nuclear material. Typically, the International Atomic Energy Agency (IAEA) relies on both neutron and gamma raybased NDA techniques for verification measurements. These verifications can be either qualitative or quantitative. Belian et al. described the techniques used to calibrate NDA instrumentation using Monte Carlo (MC) methods and reported the outcome of these calibrations [39]. Peter et al. outlined the traditional safeguards instrumentation approach and efforts that aim at verifying the correctness of information about declared nuclear materials and facilities, mainly including materials accountancy, materials composition measurements, and containment verification [40]. The traditional measurement technologies for detection of declared materials consist of NDA, which include primarily neutron and gamma measurement methods, Unattended Radiation Monitoring Systems (URMSs), other measurement and monitoring systems. LaFleur et al. presented an NDA technique called Self-Interrogation Neutron Resonance Densitometry (SINRD) which is currently being developed at Los Alamos National Laboratory (LANL) to improve existing nuclear safeguards measurements for Light Water Reactor (LWR) fuel assemblies [41]. East et al. described four systems developed at the Los Alamos Scientific Laboratory (LASL) for nondestructive analysis of nuclear fuel materials [42]. They utilized either minicomputers or a programmable calculator for data analysis and measurement control.

An important issue in nuclear safeguards is verification of operator-declared data of spent nuclear fuel. Various techniques have been assigned for this purpose. A nondestructive approach is used in the measurement of gamma radiation from spent nuclear fuel assemblies. By this technique, parameters such as burnup and cooling time can be 
calculated or verified. Willman et al. utilized gamma rays from ${ }^{137} \mathrm{Cs},{ }^{134} \mathrm{Cs}$, and ${ }^{154} \mathrm{Eu}$ to determine the consistency of operator-declared information [43]. Khusainov et al. developed a portable gamma spectrometer with a unique, near room TS detector to close this gap [44]. Swoboda et al. investigated a new spectral gamma detector for potential use in hand-held radioisotope identification devices. The socalled standard $\mathrm{Na} / \mathrm{I}$ detector is compared with other new scintillation and room TS detectors [45]. There has been a long-standing need for a room-temperature gamma-ray detector system with high efficiency and adequate resolution for nuclear safeguards applications [46]. The HPXe detector offers resolution, efficiency, intrinsic radiation resistance, and temperature stability, which can be a viable candidate for extensive application in nuclear safeguards. Wang et al. utilized two plutonium samples of known isotopic content and a broad range of gamma-ray uranium standards to show the gamma ray information from suppressed and one planar High Purity Germanium (HPGe) detectors [47].

Active nuclear safeguards measurements that rely on the time correlation between fast gamma rays and neutrons radioactive from the same fission are becoming a useful technique. Marseguerra et al. reported the results of using a ${ }^{252} \mathrm{Cf}$ source for the calibration of a plastic scintillator in nuclear safeguards experiments [48]. Pozzi and Segovia presented a method to estimate the mass of uranium oxide samples based on ${ }^{252} \mathrm{Cf}$ source-driven noise-analysis measurements [49]. The experiments results showed that the mass of the samples can be successfully predicted using a genetic programming algorithm. Vermaercke et al. and Frazzini discussed a highly rapid and precise measurement of the plutonium and uranium content of nuclear materials, respectively $[50,51]$. The instrumentation includes a constant-current coulometric titrator and gravimetric titrator for uranium measurements and a controlled-potential coulometry system for plutonium measurements. They invented Probabilistic Safety Assessment (PSA) that has proved to be an effective method for safety analysis and risk-based decisions [52]. Lu and Jiang provided an overview of PSA applications in three areas of digital Instrumentation and Control (IC) systems in nuclear power plants [53]. Radiation monitoring is one of the diagnosis methods for nuclear safeguards measurements which is used to protect against the theft of Special Nuclear Materials (SNM) by pedestrians departing from SNM access areas [54]. Letourneau et al. reviewed the Nucifer detector which is interested in developing new safeguard techniques for next generation new reactors [55]. The large flux of neutrinos that leaves a nuclear reactor carries information about two quantities of interest for nuclear safeguard for reactor power and fissile inventory [56]. Cabrera-Palmer et al. have demonstrated the antineutrino-based nuclear reactor monitoring was feasible when using a relatively small cubic scale detector made of gadolinium loaded liquid scintillator at tens of meters standoff from a Pressurized Water Reactor (PWR) [57]. The sketch is shown in Figure 5. Parsons and Wells tested and developed a tamper-resistant liquid level instrumentation system for safeguards [58]. Their testing demonstrated the accuracy of liquid level measurement using Time Domain Reflectometry (TDR) techniques

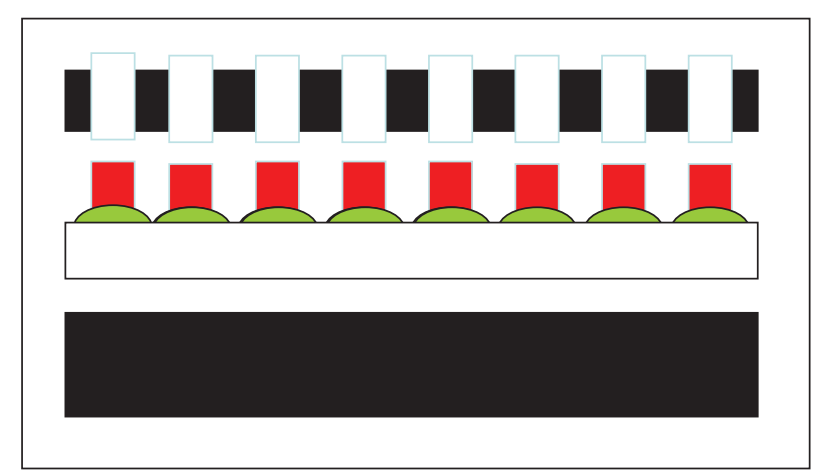

Figure 5: A schematic of the pressurized water Cherenkov detector.

and the accuracy of differential temperature and pressure measurements utilizing a custom-designed liquid level sensor probe.

\section{Nuclear Accident, after Action, and Analysis}

Nuclear accident is bloodcurdling for humanity, so we should positively prevent the nuclear accidents and analyse the damage caused by nuclear accident. There are different measuring devices and methods used for diagnosing accidental conditions, monitoring and alarming of nuclear equipment in accidental conditions, and analysis of nuclear accident. Instruments and measurements methods consist of Support Vector Classification (SVC), Group Method of Data Handling (GMDH), Support Vector Machines (SVM), Support Vector Regression (SVR), Probabilistic Neural Network (PNN), Back Propagation Network Algorithm (BPNA), Alarm Filtering and Diagnostic System (AFDS), Gamma Spectrometry (GS), Accelerator Mass Spectrometry (AMS), Solid State Nuclear Track Detectors (SSNTD), and so forth.

As a means of effectively managing severe accidents at nuclear power plants, it is important to identify and diagnose accident initiating events within a short time interval [59]. The main objective of many systems was the diagnosis of the Loss of Coolant Accidents (LOCA) which uses artificial intelligence techniques, such as GMDH and SVC. Neutron Radiography (NR) was applied for investigations of control rod behaviour and nuclear fuel cladding during steam oxidation at temperatures between 1123 and $1673 \mathrm{~K}$ under severe nuclear accident conditions [60]. Nuclear plant operators are provided with only partial information during the accident or they may have insufficient time to analyze the data despite being provided with considerable information, so it is very difficult for operators to predict the progression of LOCA. Na et al. used SVM to identify the break location of an LOCA and predict the break size using the SVC and SVR [61]. Na et al. designed a PNN that has been applied well to the classification problems in order to classify accidents into groups of original events such as LOCA, Station Blackout (SBO), Total Loss of Feedwater (TLOFW), and Steam Generator Tube Rupture (SGTR). They also designed a Fuzzy Neural Network (FNN) to identify their 


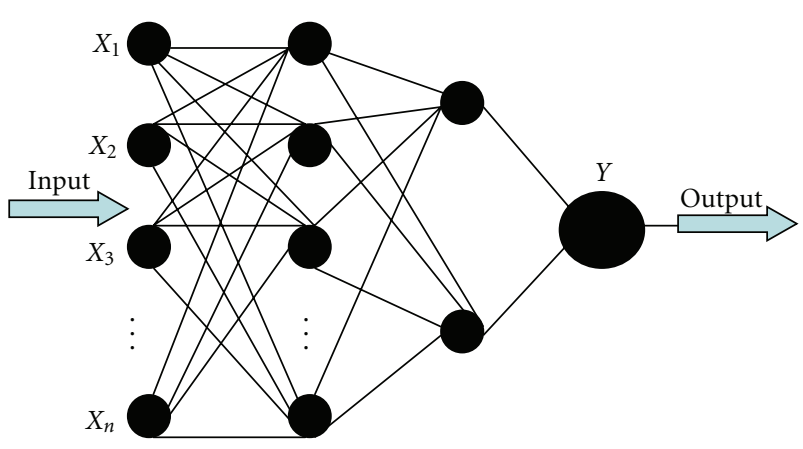

FIgURE 6: The probabilistic neural network.

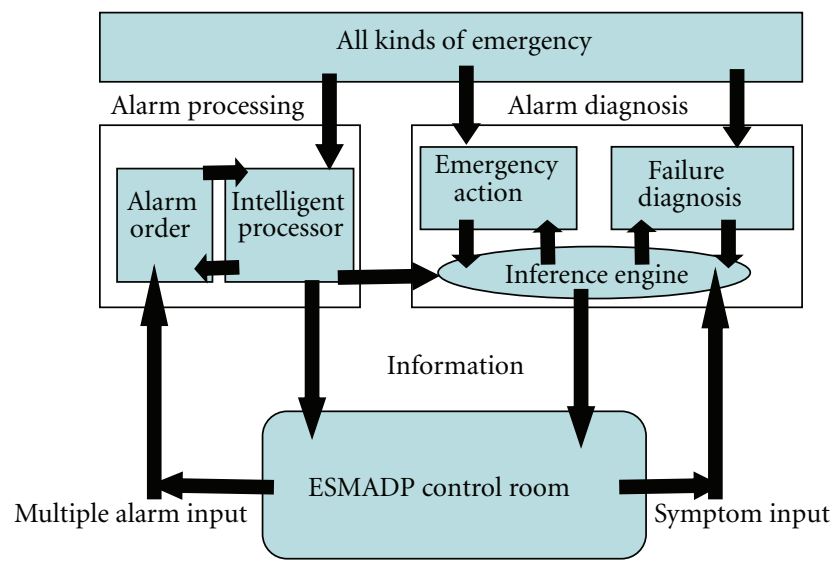

FIGURE 7: The functional structure of ESMADP.

major serious accident scenarios after the initiating events [62]. The sketch is shown in Figure 6.

Recently, the Fukushima accident showed the Station Blackout (SBO) is an important cause of core damage severe accident and radiological releases [63]. Patterson and Sc described the alarm analysis called Digital Computer System (DCS) which has been installed and is now operating in nuclear power reactor at Oldbury [64]. Cheon et al. reported the feasibility study of multiple alarm diagnosis and processing system using Neural Networks (NNs) [65]. The BPNA is applied to the training of multiple alarm patterns for the identification of faults in a Reactor Coolant Pump (RCP) system. The general mapping capability of the $\mathrm{NN}$ enables to identify a fault easily. An on-line fuzzy expert system, called AFDS, was described to provide the operators with vivid alarm photograph and system extensive failure information during anomalous states through alarm diagnosis and filtering [66]. Cheon et al. described the development strategies of a prototype expert system, called Expert System for Multiple Alarm Diagnosis and Processing (ESMADP) for multiple alarm diagnosis and processing in nuclear power plants [67]. The sketch is shown in Figure 7. This system is designed to assist operators to diagnose the nuclear power plant malfunction and to identify a dominating causal alarm among multiple fired alarms quickly.

The release of Fission Products (FPs) plays a critical role in the source term of the environment FP from a Pressurized
Water Reactor (PWR) during a hypothetical severe accident is a major target in nuclear reactor safety assessment. Gamma spectrometry is the main tool used to quantify FP releases from environment. Ducros et al. applied the on-line gamma spectrometry equipment to investigate fuel samples and measure FP release kinetics during the experiments, and the gamma scanning bench devoted to calculate the FP balances after the test [68]. Mitev et al. reported the results from measurements of ${ }^{131} \mathrm{I},{ }^{134} \mathrm{Cs}$, and ${ }^{137} \mathrm{Cs}$ of environmental samples after the Fukushima accident in Bulgaria [69]. Measurements were performed by high volume air sampling on glass fiber aerosol filters the consequence showed ${ }^{131} \mathrm{I}$ concentrations are in the range of $31-2240 \mathrm{ut} \mathrm{Bq} / \mathrm{m}^{3},{ }^{134} \mathrm{Cs}$ and ${ }^{137} \mathrm{Cs}$ concentrations are in the range of $33-456 \mathrm{ut} \mathrm{Bq} / \mathrm{m}^{3}$ and $38-637 \mathrm{ut} \mathrm{Bq} / \mathrm{m}^{3}$, respectively. Also, Endo et al. carried out soil sampling consequence at an early stage of the Fukushima Dai-ichi Nuclear Power Plant (FDNPP) accident [70]. They used Ge detector to identify and measure radioactive contaminants in soil including ${ }^{129 \mathrm{~m}} \mathrm{Te},{ }^{129} \mathrm{Te}$, ${ }^{131} \mathrm{I},{ }^{132} \mathrm{Te},{ }^{132} \mathrm{I},{ }^{134} \mathrm{Cs},{ }^{136} \mathrm{Cs},{ }^{137} \mathrm{Cs},{ }^{140} \mathrm{Ba}$, and ${ }^{140} \mathrm{La}$. The consequence showed large amounts of radioactive substances were released into the environment after the Fukushima nuclear accident. Amano et al. collected daily samples of air and tap water starting directly after the Fukushima nuclear incident and measured their radioactivity by gamma spectrometry [71]. Perrot et al. performed the low background gamma spectrometry measurements ${ }^{137} \mathrm{Cs},{ }^{131} \mathrm{I}$ and ${ }^{134} \mathrm{I}$ activities with HPGe detectors at the prisna platform located at the Centre d'Etudes Nucléaires de Bordeaux Gradignan (CENBG) laboratory in Bordeaux, France [72].

The Chernobyl nuclear reactor accident which occurred on April 26, 1986 is well known to have injected into the atmosphere a pulse of a large number of radionuclides. Paul et al. measured the concentrations of the long-lived radionuclide ${ }^{129} \mathrm{I}$ in environmental samples collected in Israel and Europe after the nuclear reactor accident [73]. The measurements were performed by Rehovot Pelletron Accelerator (RPA) and Accelerator Mass Spectrometry (AMS). Gasparro et al. described $\gamma$-ray spectrometry measurements undertaken in a range of underground laboratories for the purpose of measuring ${ }^{60} \mathrm{Co}$ more than 2 years after the Chernobyl nuclear reactor criticality event [74]. The measurements consequence showed that neutron fluence determined from ${ }^{60} \mathrm{Co}$ activity is in agreement with previous measurements using the short-lived radionuclides ${ }^{59} \mathrm{Fe}$ and ${ }^{51} \mathrm{Cr}$. Nikolaev applied many track methods and devices to investigate the conduct after Chernobyl nuclear reactor radiation accidents. They used SSNTD for the measurements of contamination in air, water, soil, biological and other objects of $\alpha$-ray radionuclides, as well as for estimation of neutron doses by means of accident dosimeters [75]. In addition, Bernhardsson et al. discussed the variations and differences of the internal and external radiation exposure of the inhabitants of several villages in the Bryansk region of Russia nearby Chernobyl [76].

\section{Conclusions}

In this paper, the advanced measurement methods and instrumentation for nuclear installations are reviewed. 
The development of the measuring (instrumentation) methods for nuclear installations and the applications of these instruments and methods are presented. Various instruments and measurement methods used in all aspects of nuclear technology, that is, nuclear power reactors, nuclear fuel cycle, safeguards and homeland security, nuclear accident, after action, and analysis, are discussed in detail. The instruments mainly include all kinds of detectors, thermometers, counters, thermocouples, spectrometers, fiber sensors, chambers, and calorimeter. The measurement methods mostly consist of various noncontact measurement methods based on optics, electrons, and magnetics.

\section{Acknowledgments}

This work was supported by the National Natural Science Foundation of China (11272368), by the Natural Science Foundation Project of CQ CSTC (2010BB2087), and by the Natural Science Foundation Project of Chongqing Education Commission (CQEC, KJ110822).

\section{References}

[1] C. Guerrero, E. Berthoumieux, D. Cano-Ott, F. Gunsing, E. Mendoza, and S. Andriamonje, "A new set-up for the simultaneous measurement of neutro-induced capture and fission reactions," in Proceedings of the 2nd International Conference on Advancements in Nuclear Instrumentation Measurement Methods and Their Applications (ANIMMA'11), Ghent, Belgium, June 2011.

[2] I. Bolshakova, V. Chekanov, I. Duran et al., "Methods and instrumentation for investigating hall sensors during their irradiation in nuclear research reactors," in Proceedings of the 1st International Conference on Advancements in Nuclear Instrumentation, Measurement Methods and Their Applications (ANIMMA'09), Marseille, France, June 2009.

[3] R. I. Scherpelz and J. E. Tanner, "Neutron measurements at nuclear power reactors [55]," Nuclear Instruments and Methods in Physics Research A, vol. 476, no. 1-2, pp. 400-404, 2002.

[4] D. Fourmentel, J.-F. Villard, A. Lyoussi et al., "Combined analysis of neutron and photon flux measurements for the Jules Horowitz reactor core mapping," in Proceedings of the 2nd International Conference on Advancements in Nuclear Instrumentation, Measurement Methods and Their Applications (ANIMMA'11), Ghent, Belgium, June 2011.

[5] H. G. Brixy, "Temperature measurement in nuclear reactors by noise thermometry," Nuclear Instruments and Methods, vol. 97, no. 1, pp. 75-80, 1971.

[6] T. Bily and L. Sklenka, "Neutronic design of instrumentation for thermal effects measurement on VR-1 reactor," in Proceedings of the 1st International Conference on Advancements in Nuclear Instrumentation, Measurement Methods and Their Applications (ANIMMA'09), Marseille, France, June 2009.

[7] J. L. Rempe, D. L. Knudson, J. E. Daw et al., "Enhanced in-pile instrumentation at the advanced test reactor," in Proceedings of the 2nd International Conference on Advancements in Nuclear Instrumentation, Measurement Methods and Their Applications (ANIMMA'11), Ghent, Belgium, June 2011.

[8] A. Porta, V. M. Bui, M. Cribier et al., "Reactor neutrino detection for non-proliferation with the NUCIFER experiment," IEEE Transactions on Nuclear Science, vol. 57, no. 5, pp. 27322739, 2010.
[9] J. F. Villard, S. Fourrez, D. Fourmentel, and A. Legrand, "Improving high-temperature measurements in nuclear reactors with $\mathrm{Mo} / \mathrm{Nb}$ thermocouples," International Journal of Thermophysics, vol. 29, no. 5, pp. 1848-1857, 2008.

[10] G. Cheymol, B. Brichard, and J. F. Villard, "Fibre optics for metrology in nuclear research reactors applications to dimensional measurements," in Proceedings of the 1st International Conference on Advancements in Nuclear Instrumentation, Measurement Methods and Their Applications (ANIMMA'09), Marseille, France, June 2009.

[11] G. Y. Buimistriuc and A. M. Rogov, "Intelligent fiber optic pressure sensor for measurements in extreme conditions," in Proceedings of the 1st International Conference on Advancements in Nuclear Instrumentation, Measurement Methods and Their Applications (ANIMMA'09), Marseille, France, June 2009.

[12] C. Papastefanou, "Measurement of neutron flux and albedo of water for thermal neutrons with foils of indium in a subcritical nuclear reactor," Journal of Radioanalytical and Nuclear Chemistry, vol. 261, no. 3, pp. 671-678, 2004.

[13] Y. G. Li, Y. Q. Shi, Y. B. Zhang, and P. Xia, "Reactor fission rate measurement for miniature neutron source reactor by solid state nuclear track detector," Radiation Measurements, vol. 34, no. 1-6, pp. 589-591, 2001.

[14] M. Laurie, M. A. Fütterer, J. M. Lapetite, S. Fourrez, and R. MoriceNew, "new temperature monitoring devices for hightemperature irradiation experiments in the high flux reactor petten," Nuclear Science, vol. 58, pp. 2319-2324, 2011.

[15] N. Khentout and B. Meftah, "Design and realization of a measurement system of cold neutron speeds by TOF method," in Proceedings of the 1st International Conference on Advancements in Nuclear Instrumentation, Measurement Methods and Their Applications (ANIMMA'09), Marseille, France, June 2009.

[16] A. K. Bhattacharya, J. T. Verdeyen, F. T. Adler, and L. Goldstein, "Measurement of the local radiation field in a nuclear reactor by microwave interferometric techniques," Nuclear Instruments and Methods, vol. 95, no. 2, pp. 313-325, 1971.

[17] O. Meirav, M. Adam, E. Boaretto et al., "Measurements of ${ }^{129} \mathrm{I}$ in a nuclear power reactor by accelerator mass spectrometry," International Journal of Radiation Applications and Instrumentation A, vol. 43, no. 11, pp. 1420-1424, 1992.

[18] R. Coulon, S. Normand, G. Ban et al., "Sodium fast reactor power monitoring using ${ }^{20} \mathrm{~F}$ tagging agent," in Proceedings of the 1st International Conference on Advancements in Nuclear Instrumentation, Measurement Methods and Their Applications (ANIMMA'09), Marseille, France, June 2009.

[19] A. K. Sang, M. E. Froggatt, D. K. Gifford, S. T. Kreger, and B. D. Dickerson, "One centimeter spatial resolution temperature measurements in a nuclear reactor using Rayleigh scatter in optical fiber," IEEE Sensors Journal, vol. 8, no. 7, pp. 13751380, 2008.

[20] L. K. Yarovoi, L. Y. Robur, A. V. Gnatovsky, and E. P. Vdalov, "holographic equipment for testing the construction of the nuclear power plant," in Proceedings of the 4th Regional Meeting, 1997.

[21] S. D. Eastham, D. J. Coates, and G. T. Parks, "A novel method for rapid comparative quantitative analysis of nuclear fuel cycles," Annals of Nuclear Energy, vol. 42, pp. 80-88, 2012.

[22] M. H. Noiré, L. Couston, E. Douarre, D. Pouyat, C. Bouzon, and P. Marty, "A new sol-gel derived optical fiber sensor for high acidity measurements: application in nuclear fuel reprocessing," Journal of Sol-Gel Science and Technology, vol. 17, no. 2, pp. 131-136, 2000. 
[23] I. Matsson, B. Grapengiesser, and B. Andersson, "LOKET-a gamma-ray spectroscopy system for in-pool measurements of thermal power distribution in irradiated nuclear fuel," Nuclear Instruments and Methods in Physics Research A, vol. 569, no. 3, pp. 872-882, 2006.

[24] L. Viererbl, Z. Lahodová, A. Voljanskij et al., "Measurement of gamma and neutron radiations inside spent fuel assemblies with passive detectors," Nuclear Instruments and Methods in Physics Research A, vol. 652, no. 1, pp. 90-93, 2011.

[25] M. Battaglieri, R. de Vita, G. Firpo et al., "An anti-neutrino detector to monitor nuclear reactor's power and fuel composition," Nuclear Instruments and Methods in Physics Research A, vol. 617, no. 1-3, pp. 209-213, 2010.

[26] P. F. Peck, "Control analysis of ${ }^{235} \mathrm{U}$ in nuclear fuel by a neutron absorption method," Journal of Radioanalytical Chemistry, vol. 48, no. 1-2, pp. 73-82, 1979.

[27] G. Perret and K. A. Jordan, "On the combination of delayed neutron and delayed gamma techniques for fission rate measurement in nuclear fuel," in Proceedings of the 2nd International Conference on Advancements in Nuclear Instrumentation, Measurement Methods and Their Applications (ANIMMA'11), Ghent, Belgium, June 2011.

[28] X. Y. Liu, D. A. Andersson, and B. P. Uberuaga, "Firstprinciples DFT modeling of nuclear fuel materials," Journal of Materials Science, vol. 47, no. 21, pp. 7367-7384, 2012.

[29] E. Rosenkrantz, J. Y. Ferrandis, G. Leveque, and D. Baron, "Ultrasonic measurement of gas pressure and composition for nuclear fuel rods," Nuclear Instruments and Methods in Physics Research A, vol. 603, no. 3, pp. 504-509, 2009.

[30] S. Subbuthai, P. Sahoo, R. Ananthanarayanan, A. Nageswara Rao, and R. V. Subba Rao, "Feasibility studies for the detection of third phase during reprocessing of fast reactor fuel," Journal of Radioanalytical and Nuclear Chemistry, vol. 291, no. 2, pp. 879-883, 2012.

[31] T. Kamei and A. Serizawa, "Measurement of 2-dimensional local instantaneous liquid film thickness around simulated nuclear fuel rod by ultrasonic transmission technique," Nuclear Engineering and Design, vol. 184, no. 2-3, pp. 349-362, 1998.

[32] T. Inoue, "Actinide recycling by pyro-process with metal fuel FBR for future nuclear fuel cycle system," Progress in Nuclear Energy, vol. 40, no. 3-4, pp. 547-554, 2002.

[33] S. Normand, V. Kondrasov, G. Corre, and C. Passard, "PING: a new approach for nuclear fuel cycle instrumentation," in Proceedings of the 1st International Conference on Advancements in Nuclear Instrumentation, Measurement Methods and Their Applications (ANIMMA'09), Marseille, France, June 2009.

[34] I. Badawy and W. A. EI-Gammal, "Measurement of low enriched uranium fuel pellets," in Proceedings of the 16th National Conference on Radio Science, Cairo, Egypt, February 1999.

[35] A. Gavron, L. E. Smith, and J. J. Ressler, "Analysis of spent fuel assemblies using a lead slowing down spectrometer," Nuclear Instruments and Methods in Physics Research A, vol. 602, no. 2, pp. 581-587, 2009.

[36] S. K. Das, C. S. Kedari, and S. C. Tripathi, "Spectrophotometric determination of trace amount of uranium (VI) in different aqueous and organic streams of nuclear fuel processing using 2-(5-bromo-2-pyridylazo-5-diethylaminophenol)," Journal of Radioanalytical and Nuclear Chemistry, vol. 285, no. 3, pp. 675-681, 2010.

[37] A. I. Hawari, J. Chen, B. J. Su, and Z. X. Zhao, "Assessment of on-line burnup monitoring of pebble bed reactor fuel using passive gamma-ray spectrometry," IEEE Transactions on Nuclear Science, vol. 49, no. 3, pp. 1249-1253, 2002.
[38] U. Meier, J. Heinze, L. Lange, C. Hassa, L. Rackwitz, and T. Doerr, "Characterisation of the combustion performance of low emission fuel injectors with laser measurements," CEAS Aeronautical Journal, vol. 3, no. 1, pp. 45-53, 2012.

[39] A. P. Belian, L. C. Bourva, S. C. Kane, and A. R. Lebrun, "The use of Monte Carlo techniques for the calibration of IAEA safeguards instrumentation," in Proceedings of the 1st International Conference on Advancements in Nuclear Instrumentation, Measurement Methods and Their Applications (ANIMMA'09), Marseille, France, June 2009.

[40] N. Peter, M. Koskelo, and M. Stein, "The role of the nuclear industry in advancing safeguards instrumentation," in Proceedings of the 1st International Conference on Advancements in Nuclear Instrumentation, Measurement Methods and Their Applications (ANIMMA'09), Marseille, France, June 2009.

[41] A. M. Lafleur, W. S. Charlton, H. O. Menlove, and M. T. Swinhoe, "Comparison of fresh fuel experimental measurements to MCNPX calculations using self-interrogation neutron resonance densitometry," Nuclear Instruments and Methods in Physics Research A, vol. 680, pp. 168-178, 2012.

[42] L. V. East, E. R. Martin, T. L. Atwell, R. H. Augustson, and H. O. Menlove, "Automated nondestructive assay instrumentation for nuclear materials safeguards," Nuclear Science, vol. 22, no. 1, pp. 739-743, 1975.

[43] C. Willman, A. Håkansson, O. Osifo, A. Bäcklin, and S. J. Svärd, "Nondestructive assay of spent nuclear fuel with gamma-ray spectroscopy," Annals of Nuclear Energy, vol. 33, no. 5, pp. 427-438, 2006.

[44] A. K. Khusainov, T. A. Antonova, V. V. Lysenko et al., "High resolution, portable gamma spectrometer not requiring liquid nitrogen cooling for field use for nuclear security and safeguards applications," in Proceedings of the Nuclear Science Symposium Conference, Dresden, Germany, October 2008.

[45] M. Swoboda, R. Arlt, V. Gostilo et al., "Spectral gamma detectors for hand-held radioisotope identification devices (RIDs) for nuclear security applications," in Proceedings of the Nuclear Science Symposium, Medical Imaging Conference, Symposium on Nuclear Power Systems and the 14th International Workshop on Room Temperature Semiconductor X- and Gamma-Ray Detectors, pp. 4296-4302, Rome, Italy, October 2004.

[46] D. H. Beddingfield, A. Beyerle, P. A. Russo, K. Ianakiev, D. T. Vo, and V. Dmitrenko, "High-pressure xenon ion chambers for gamma-ray spectroscopy in nuclear safeguards," Nuclear Instruments and Methods in Physics Research A, vol. 505, no. 1-2, pp. 474-477, 2003.

[47] T. F. Wang, S. M. Horne, R. A. Henderson, K. E. Roberts, and D. K. Vogt, "Exploring simultaneous single and coincident gamma-ray measurements for U/Pu assay in safeguards," in Proceedings of the 2nd International Conference on Advancements in Nuclear Instrumentation, Measurement Methods and Their Applications (ANIMMA'11), Ghent, Belgium, June 2011.

[48] M. Marseguerra, E. Padovani, and S. A. Pozzi, "Use of the MCNP-Polimi code for time-correlation safeguards measurements," Progress in Nuclear Energy, vol. 43, no. 1-4, pp. 305311, 2003.

[49] S. A. Pozzi and J. Segovia, " ${ }^{252}$ Cf source-correlated transmission measurements and genetic programming for nuclear safeguards," Nuclear Instruments and Methods in Physics Research A, vol. 491, no. 1-2, pp. 326-341, 2002.

[50] P. Vermaercke, F. F. Arboccò, L. Sneyers, A. Leal, and M. Bruggeman, "Environmental monitoring for safeguards using $\mathrm{k}_{0}$-standardised neutron activation analysis," in Proceedings of the 1st International Conference on Advancements in Nuclear Instrumentation, Measurement Methods and Their Applications (ANIMMA'09), Marseille, France, June 2009. 
[51] T. L. Frazzini, "Application of automated analytical instrumentation to the determination of uranium and plutonium for nuclear safeguards measurements," IEEE Transactions on Nuclear Science, vol. 29, no. 1, pp. 866-868, 1981.

[52] Thomas H. Smith, Ronald J. Hall, and L. Donald Williams, "Analytic methods for fuel-cycle safety studies," IEEE Transactions on Reliability, vol. R-25, no. 3, pp. 184-190, 1976.

[53] L. Lu and J. Jiang, "Probabilistic safety assessment for instrumentation and control systems in nuclear power plants: an overview," Journal of Nuclear Science and Technology, vol. 41, no. 3, pp. 323-330, 2004.

[54] P. E. Fehlaul, "Integrated neutrod gamma-ray portal monitors for nuclear safeguards," Nuclear Science, vol. 41, no. 4, pp. 922 926, 1994.

[55] A. Letourneau, V.-M. Bui, M. Cribier et al., "Nucifer: a small electron-antineutrino detector for fundamental and safeguard studies," in 2nd International Conference on Advancements in Nuclear Instrumentation, Measurement Methods and Their Applications (ANIMMA'11), Ghent, Belgium, June 2011.

[56] R. Baskaran, V. Subramanian, J. Misra, R. Indira, P. Chellapandi, and B. Raj, "Aerosol characterization and measurement techniques towards SFR safety studies," in Proceedings of the 1st International Conference on Advancements in Nuclear Instrumentation, Measurement Methods and Their Applications (ANIMMA'09), Marseille, France, June 2009.

[57] B. Cabrera-Palmer, D. Reyna, L. Sadler et al., "Advances towards readily deployable antineutrino detectors for reactor monitoring and safeguards," in Proceedings of the 1st International Conference on Advancements in Nuclear Instrumentation, Measurement Methods and Their Applications (ANIMMA'09), Marseille, France, June 2009.

[58] B. B. Parsons and J. L. Wells, "Tamper and radiation resistant instrumentation for safeguarding special nuclear material," IEEE Transactions on Nuclear Science, vol. NS-24, no. 1, pp. 616-620, 1976.

[59] S. H. Lee, Y. G. No, M. G. Na, K. I. Ahn, and S. Y. Park, "Diagnostics of loss of coolant accidents using SVC and GMDH models," IEEE Transactions on Nuclear Science, vol. 58, no. 1, pp. 267-276, 2011.

[60] M. Große, M. Steinbrück, J. Stuckert, A. Kastner, and B. Schillinger, "Application of neutron radiography to study material processes during hypothetical severe accidents in nuclear reactors," Journal of Materials Science, vol. 47, no. 18, pp. 6505-6512, 2012.

[61] M. G. Na, W. S. Park, and D. H. Lim, "Detection and diagnostics of loss of coolant accidents using support vector machines," IEEE Transactions on Nuclear Science, vol. 55, no. 1, pp. 628-636, 2008.

[62] M. G. Na, S. H. Shin, S. M. Lee et al., "Prediction of major transient scenarios for severe accidents of nuclear power plants," IEEE Transactions on Nuclear Science, vol. 51, no. 2, pp. 313-321, 2004.

[63] W. K. Lu and B. Zhao, "Preliminary evaluation of the effect of several management measures for the station blackout severe accident of the pressurized water reactor nuclear power plant," in Proceedings of the International Conference on Information Systems for Crisis Response and Management (ISCRAM'11), pp. 455-460, Harbin, China, November 2011.

[64] D. Patterson, "Application of a computerised alarm-analysis system to a nuclear power station," Electrical Engineers, vol. 115, no. 12, pp. 1858-1864, 1968.

[65] S. W. Cheon, S. H. Chang, H. Y. Chung, and Z. N. Bien, "Application of neural networks to multiple alarm processing and diagnosis in nuclear power plants," IEEE Transactions on Nuclear Science, vol. 40, no. 1, pp. 11-20, 1993.
[66] S. S. Choi, K. S. Kang, H. G. Kim, and S. H. Chang, "Development of an on-line fuzzy expert system for integrated alarm processing in nuclear power plants," IEEE Transactions on Nuclear Science, vol. 42, no. 4, pp. 1406-1418, 1995.

[67] S. W. Cheon, S. H. Chang, and H. Y. Chung, "Development strategies of an expert system for multiple alarm processing and diagnosis in nuclear power plants," IEEE Transactions on Nuclear Science, vol. 40, no. 1, pp. 21-30, 1993.

[68] G. Ducros, S. Bernard, M. P. Ferroud-Plattet, and O. Ichim, "Use of gamma spectrometry for measuring fission product releases during a simulated PWR severe accident: application to the VERDON experimental program," in Proceedings of the 1st International Conference on Advancements in Nuclear Instrumentation, Measurement Methods and Their Applications (ANIMMA'09), Marseille, France, June 2009.

[69] K. Mitev, R. T. sibranski, V. Avramov et al., "Measurements of ${ }^{133} \mathrm{I},{ }^{134} \mathrm{Cs}$ and ${ }^{137} \mathrm{Cs}$ In environmental samples in Bulgaria after the Fukushima accident," in Proceedings of the Nuclear Science Symposium and Medical Imaging Conference, Valencia, Spain, October 2011.

[70] S. Endo, S. Kimura, T. Takatsuji, K. Nanasawa, T. Imanaka, and K. Shizuma, "Measurement of soil contamination by radionuclides due to the Fukushima Dai-ichi nuclear power plant accident and associated estimated cumulative external dose estimation," Journal of Environmental Radioactivity, vol. 111, pp. 18-27, 2012.

[71] H. Amano, M. Akiyama, B. Chunlei et al., "Radiation measurements in the Chiba Metropolitan Area and radiological aspects of fallout from the Fukushima Dai-ichi nuclear power plants accident," Journal of Environmental Radioactivity, vol. 111, pp. 42-52, 2012.

[72] F. Perrot, P. Hubert, C. Marquet et al., "Evidence of ${ }^{131} \mathrm{I}$ and ${ }^{134,137}$ Cs activities in Bordeaux, France due to the Fukushima nuclear accident," Journal of Environmental Radioactivity, vol. 114, pp. 61-65, 2012.

[73] M. Paul, D. Fink, G. Hollos, A. Kaufman, W. Kutschera, and M. Magaritz, "Measurement of 129I concentrations in the environment after the Chernobyl reactor accident," Nuclear Instruments and Methods in Physics Research B, vol. 29, no. 1-2, pp. 341-345, 1987.

[74] J. Gasparro, M. Hult, K. Komura et al., "Measurements of ${ }^{60} \mathrm{Co}$ in spoons activated by neutrons during the JCO criticality accident at Tokai-mura in 1999," Journal of Environmental Radioactivity, vol. 73, no. 3, pp. 307-321, 2004.

[75] V. A. Nikolaev, "Application of SSNTDs to investigations of radiation accident after-effects," Radiation Measurements, vol. 25, no. 1-4, pp. 337-345, 1995.

[76] C. Bernhardsson, I. Zvonova, C. Rääf, and S. Mattsson, "Measurements of long-term external and internal radiation exposure of inhabitants of some villages of the Bryansk region of Russia after the Chernobyl accident," Science of the Total Environment, vol. 409, no. 22, pp. 4811-4817, 2011. 

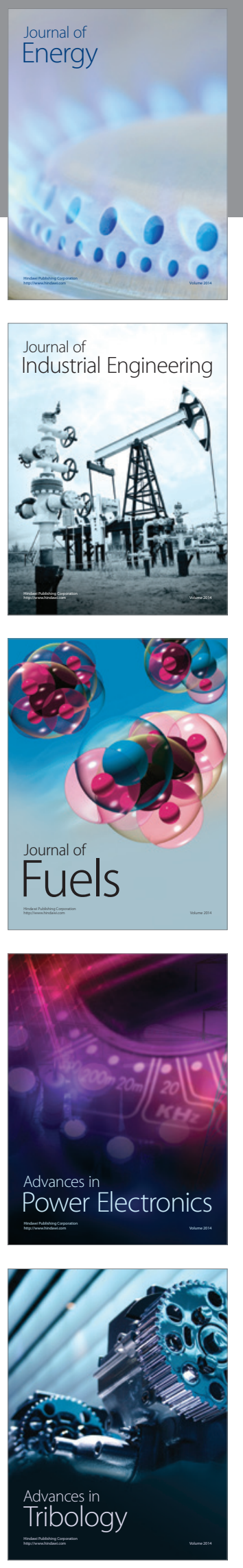
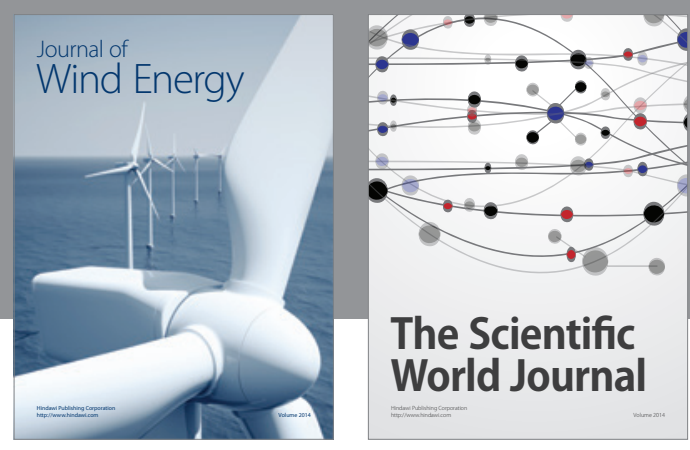

The Scientific World Journal

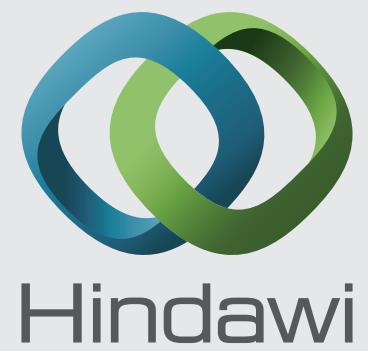

Submit your manuscripts at http://www.hindawi.com
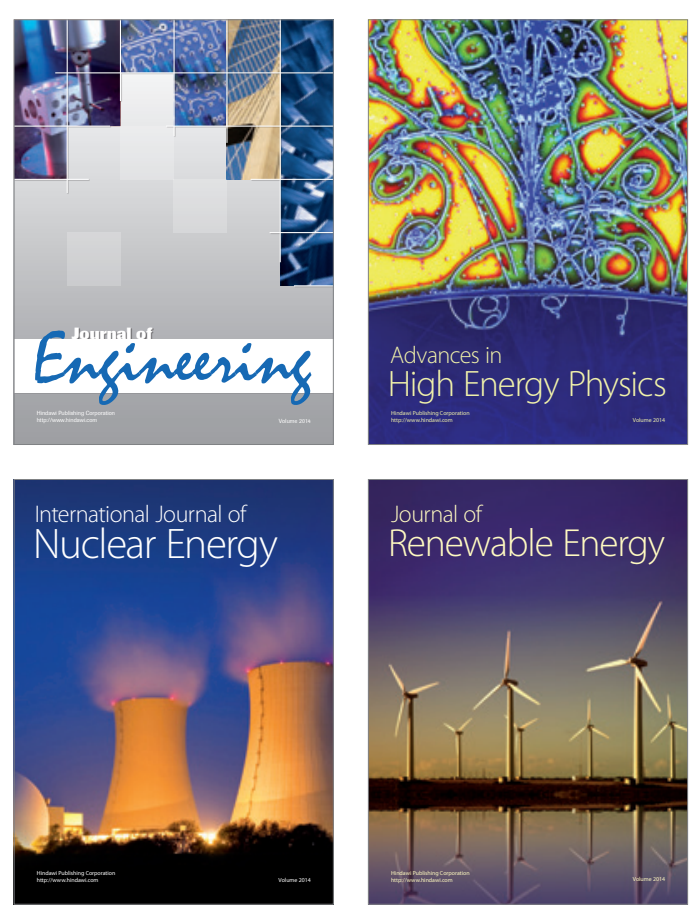

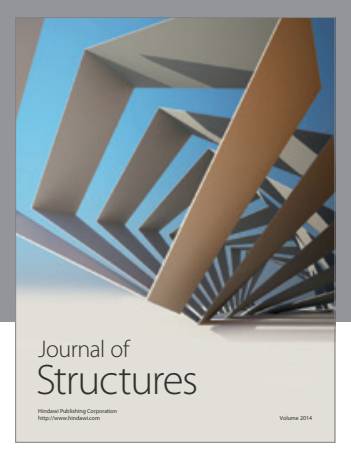

Rotating
Mechinery
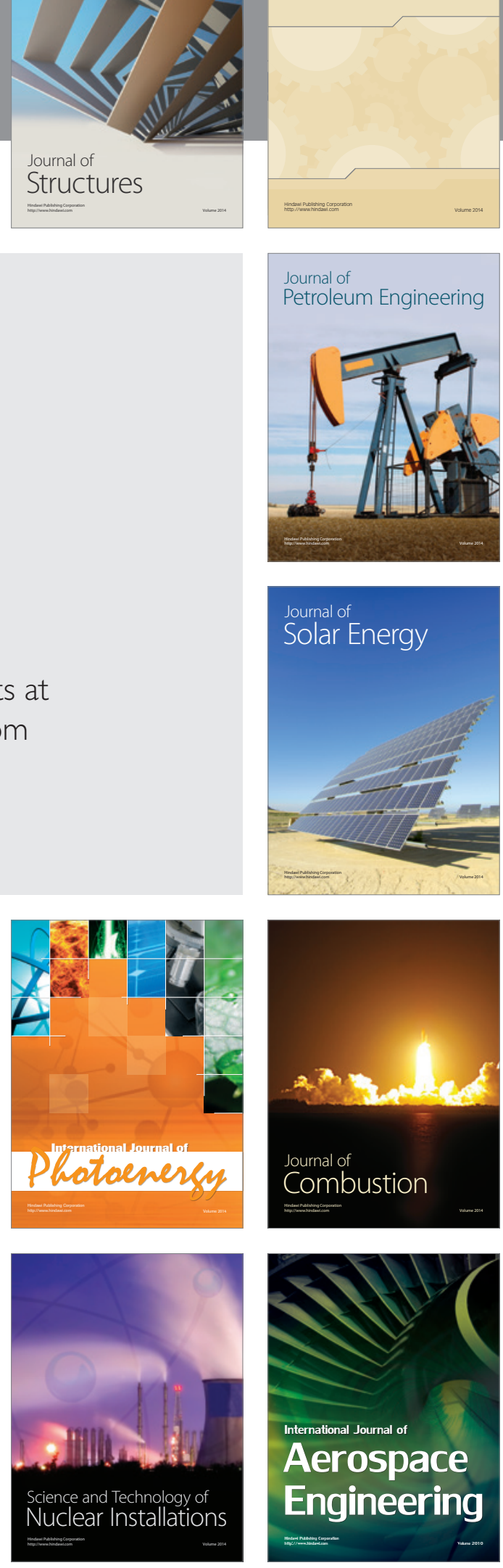